\title{
Modelling at Nanoscale
}

\author{
Paolo Di Sia
}

Additional information is available at the end of the chapter

http://dx.doi.org/10.5772/50755

\section{Introduction}

One of the most important aspects at nanoscale concerns the charge transport, which can be influenced by particles dimensions and assumes different characteristics with respect to those of bulk. In particular, if the mean free path of charges, due to scattering phenomena, is larger than the particle dimensions, we have a mesoscopic system, in which the transport depends on dimensions and in principle it is possible to correct the transport bulk theories by considering this phenomenon. A similar situation occurs also in a thin film, in which the smallest nanostructure dimension can be less than the free displacement and therefore requires variations to existing theoretical transport bulk models. Therefore a rigorous knowledge of transport properties is to be acquired. From a theoretical viewpoint, various techniques can be used for the comprehension of transport phenomena, in particular analytical descriptions based on transport equations. Many existing and used theories at today regard numerical approaches, not offering analytical results, which would be of great mathematical interest and in every case suitable to be implemented through the experimental data existing in literature and continuously found by the experimentalists.

To establish the applicability limit of a bulk model and to investigate the time response of systems at nanoscale, recently it has appeared a novel theoretical approach, based on correlation functions obtained by a complete Fourier transform of the frequency-dependent conductivity of the studied system [1]. With this model it is possible to calculate exactly the expressions of the most important connected functions, i.e. the velocities correlation function, the mean free displacement and the diffusion coefficient.

At nanoscale we are in the middle between classical and quantum effects, macroscopic and microscopic properties of the matter [2]. Actually one of the most important experimental technique for the study of the frequency-dependent complex-valued far-infrared photoconductivity $\sigma(\omega)$ is the Time-resolved THz Spectroscopy (TRTS), an ultrafast noncontact optical probe; data are normally fitted via Drude-Lorentz, Drude-Smith or effective medium models [3]. The quantum effects in the nanoworld have opened new ways in a lot of old and new technological sectors. 
In the following it is illustrated an overview of the fundamental models that, starting from the Drude model, have attempted to analyze and explain in increasing accuracy the transport phenomena of the matter at solid state level and in particular at nanoscale, until the recent developments concerning the variations of Drude-Lorentz-like models, which involve in particular the concept of plasmon.

\section{The Drude model}

The most meaningful characteristics of metals are their elevated properties of electric and thermal conduction. Over the years this fact has brought to think in terms of a model in which the electrons are relatively free and can move under the influence of electric fields. Historically two models of the elementary theory of metals are born:

The Drude model, published in 1900 and based on the kinetic theory of an electron gas in a solid. It is assumed that all the electrons have the same average kinetic energy $E_{m}$;

The previous model integrated with the foundations of quantum mechanics, called Sommerfeld model.

In the Drude model the valence electrons of atoms are considered free inside the metal; all the electrons move as an electronic gas. It is assumed that all the electrons have the same energy $E_{m}$ and that it exists a mechanism of collisions among ions and electrons, allowing the thermal equilibrium for the electrons; this fact implies the application of the kinetic theory of gases to such electronic gas. Drude published the theory three years after the discovery of the electron from J. J. Thomson. The free electrons have only kinetic energy, not potential energy; the average energy $E_{m}$ is therefore $(3 / 2) k_{B} T$. It can be correlated to an average quadratic speed $v_{m}$ from the relation $E_{m}=(3 / 2) k_{B} T=m v_{m}^{2} / 2$, denoting $m$ the mass of the free electron. At environment temperature it is $v_{m} \cong 10^{7} \mathrm{~cm} / \mathrm{s}$, representing the average thermal speed of the electrons. It was assumed also that the electrons have collisions as instant events, i.e. the time of the diffraction is very smaller with respect to every other considered time. Through such collisions, the electrons acquire a thermal equilibrium corresponding to the temperature $T$ of the metal. If the electrons don't collide, they move in linear way following the Newton laws. The presence of a constant electric field determines an extra average velocity (the drift velocity) given by $v_{d}=-(e E / m) t$. The relaxation time $\tau$ is defined as the average time between two collisions; it is so possible to get a mean free path, defined by $l_{m f p}=v_{m} \tau$. The current density is $\vec{J}=\sigma_{\text {cond }} \vec{E}$, with $\sigma_{c o n d}$ electric conductivity. This result, obtained by Drude, is an important goal of the classic theory for the conduction of metals (said "Drude theory").

\section{The Drude-Lorentz model}

The Lorentz model (1905) is a refining of the Drude model, in which the statistical aspects are specified. The electrons are considered as free charges, with charge " $e$ "; they are described by a maxwellian velocity distribution. Considering an electron gas in a spatial region with a constant electric field, the drift velocity of the electrons is constant; this 
corresponds to a current density $\vec{J}$ proportional to the applied field $\vec{J}=\sigma_{0} \vec{E}$, with $\sigma_{0}=n e^{2} \tau / m$ ( $n$ is the electron density). Estimating the relaxation time $\tau$, Drude and Lorentz have obtained values of conductivity in good accordance with the experiments. In presence of an electric field of the form $E(t)=E_{0} e^{-i \omega t}$, the complex conductivity assumes the form $\sigma_{\omega}=\sigma_{0} /(1-i \omega \tau)$. Such model, said "Drude-Lorentz model", has received great success, but has also underlined series difficulties.

\section{The most utilized Drude-Lorentz-like models}

One of the most utilized models for describing experimental transport data is the DrudeLorentz model [4,5]; with such model the main transport parameters are obtainable. Starting from the Drude-Lorentz model, it is possible to obtain the velocities correlation function, from this the quadratic average distance crossed by the charges as a function of time and examine directly the possible compatibility with the Einstein relation.

Considerable variations of this model were made in this years; the most used are the following:

1. the "Maxwell-Garnett (MG) model": in this model the dielectric function is given by a Drude term with an additional "vibrational" contribution at a finite frequency $\omega_{0}[6]$, leading to a dielectric function of the form:

$$
\varepsilon_{/ /}(\omega)=1-\frac{\omega_{p}^{2}}{\omega(\omega+i / \tau)}+\frac{\omega_{s}^{2}}{\omega_{0}^{2}-\omega^{2}-i \gamma \omega}
$$

where the amplitude $\omega_{s}$, the resonant frequency $\omega_{0}$ and the damping constant $\gamma$ are material dependent constants. The MG model usually describes an isotropic matrix containing spherical inclusions isolated from each other, such as the metal particles dispersed in a surrounding host matrix.

2. In the "effective medium theories (EMTs)" the electromagnetic interactions between pure materials and host matrices are approximately taken into account [7]. The commonly used EMTs include the Maxwell-Garnett (MG) model and the "Bruggeman (BR) model", which is a variation of the MG model.

Generally, in the THz regime, the dielectric function $\varepsilon_{m}(\omega)$ consists of contributions of the high-frequency dielectric constant, conduction free electrons, and lattice vibration [8]:

$$
\varepsilon_{m}(\omega)=\varepsilon_{\infty}-\frac{\omega_{p}{ }^{2}}{\omega^{2}+i \gamma \omega}+\sum_{j} \frac{\varepsilon_{s t_{j}} \omega_{T O_{j}}{ }^{2}}{\omega_{T O_{j}}{ }^{2}-\omega^{2}-i \Gamma_{j} \omega}
$$

in which $\varepsilon_{\infty}$ is the high-frequency dielectric constant, the second term describes the contribution of free electrons or plasmons and the last term stands for the optical phonons. 
When the response originates mainly from the contribution of free electrons or plasmons, it is usually adopted the Drude model:

$$
\varepsilon_{m}(\omega)=\varepsilon_{\infty}-\frac{\omega_{p}{ }^{2}}{\omega^{2}+i \gamma \omega}
$$

which described well the dielectric properties of metals and semiconductors [7].

If instead the interaction of a radiation field with the fundamental lattice vibration plays a dominant role and results in absorption of electromagnetic wave, due to the creation or annihilation of lattice vibration, the dielectric function $\varepsilon_{m}(\omega)$ mainly consists of the contributions of the lattice vibrations, expressed by the classical pseudo-harmonic phonon model in the first approximation [7]:

$$
\varepsilon_{m}(\omega)=\varepsilon_{\infty}+\frac{\varepsilon_{s t} \omega_{T O}{ }^{2}}{\omega_{T O}{ }^{2}-\omega^{2}-i \gamma \omega}
$$

\section{The Smith model}

Smith has started from the response theory for the optical conductivity, considering an electric field impulse applied to a system, in order to examine the answer with respect to the current. He has utilized the following Fourier transform for the frequency-dependent complex conductivity:

$$
\sigma(\omega)=\int_{0}^{\infty} j(t) \exp (i \omega t) d t
$$

A field impulse, which exceeds every other acting force on the system, permits to assume the hypothesis that the electrons initially can be considered totally free; therefore it holds:

$$
j(0)=n * e^{2} / m
$$

with $n^{*}$ effective electron density. After calculation, the real part of $\sigma(\omega)$ results:

$$
\int_{0}^{\infty} \operatorname{Re} \sigma(\omega) d \omega=\frac{\pi}{2} j(0)=\frac{\omega_{p}^{2}}{8}
$$

If the initial current decays exponentially to its initial value with relaxation time $\tau$, it is possible to write:

$$
j(t) / j(0)=\exp (-t / \tau)
$$

from which the standard Drude formula is obtainable:

$$
\sigma(\omega)=\left(n^{*} e^{2} \tau / m\right) /(1-i \omega \tau)
$$


Eq. (9) can be considered the first term of a series of the form:

$$
j(t) / j(0)=\exp (-t / \tau)\left[1+\sum_{n=1}^{\infty} c_{n}(t / \tau)^{n} / n !\right]
$$

The $c_{n}$ factors hold into account of the original electrons speed remained after the $\mathrm{n}$-th collision. The analytical form of the complex conductivity is therefore [9]:

$$
\sigma(\omega)=\frac{n^{*} e^{2} \tau}{m(1-i \omega \tau)}\left[1+\sum_{n=1}^{\infty} \frac{c_{n}}{(1-i \omega \tau)^{n}}\right]
$$

\section{Plasmonics}

\subsection{Introduction}

A lot of nanostructures, in particular the nanostructured metals, show very complex and interesting optical properties. One of the most interesting and promising phenomena encountered in these structures are electromagnetic resonances, due to collective oscillations of the conduction electrons, said "plasmons". Plasmon modes exist in different geometries and in various metals, in particular in noble metals such as gold and silver. Under determined circumstances, plasmons are excited by light; this leads to strong light scattering and absorption and to an increase of the local electromagnetic field. The interest in plasmon modes has started to the beginning of the $20^{\text {th }}$ century, with Zenneck (1907), Mie (1908), Sommerfeld (1909) and other scientists; recent advances regarding the structure, the manipulation and the observation at nanoscale, have increased the study of this scientific topic. The theoretical efforts have encountered also the growing demand at technological level, in particular for semiconductor based integrated electronic components, optical applications and new nano-components. Actually it remains an important challenge for research and development processes, like the guide of light in integrated optical systems and the interface with electronic components. A lot of nanostructures are believed to be one of the key ingredients of the future optoelectronic devices [10].

\subsection{Plasmons}

The concept of plasma is very useful in the description of some aspects of the interaction radiation/conductive matter; the free electrons of the conductive material (for example a metal) are considered as an electron fluid with high density, of order of $10^{23} \mathrm{~cm}^{-3}$. Such concept is the base of the classic Drude model, which assumes that the material contains stopped positive ions and a gas of classical not interacting electrons, whose motion results slowed by a force of viscous friction, due to the collisions with the ions, and characterized from a relaxation time $\tau$. The motion of electrons results so casual, due to the continuous collisions with the lattice. The plasma frequency is defined as the proper frequency of the collective motion of electrons in the following way: 


$$
\omega_{p}^{2}=\frac{\sigma_{0}}{\varepsilon_{0} \tau}=\frac{n e^{2}}{\varepsilon_{0} m}
$$

with $\sigma_{0}$ conductivity of material and common meaning of the other mathematical symbols. The dielectric constant of metal can be written as a function to the plasma frequency; in first approximation it results:

$$
\varepsilon(\omega)=1-\frac{\omega_{p}^{2}}{\omega^{2}}
$$

From the comparison between $\omega$ and $\omega_{p}$, we can deduce the behaviour of the electromagnetic waves arriving to the metal. If it holds $\omega<\omega_{p}$, the dielectric constant is negative, therefore its square root is imaginary pure; this involves the reflection of the incident wave. In the contrary case, the square root of the dielectric constant is real and the incident wave can propagate in the medium with a small attenuation.

The plasma oscillations are the fluctuations of charge density, which happen to the frequency $\omega_{p}$ and propagate in the metal. The quanta of such fluctuations inside the volume are the "volume plasmons".

There is also a mechanism on the metal surface, characterized by the "surface plasmons". Normally it appears in the wavelength range between the visible and the infrared for the interface air/metal. Localized surface electromagnetic excitations can exist at the surface of nanoparticles and metallic nanostructures: the "localized surface plasmons" (LSP) [11]. The frequency and the intensity of the radiation are very sensitive with respect to the dimension, form and morphology of the nanostructures [12]. Nanoparticles and nanostructures with smaller dimensions with respect to the wavelength of the exciting light are characterized by a wide absorption band, normally in the range of the visible and near infrared spectrum.

\subsection{Related theoretical models}

The models concerning plasmonics simulate the extinction of the "localized surface plasmons resonance" (LSPR) from nanoparticles and nanoarrays; the application regards the calculation of the light absorption and the scattering. The most used models are:

- $\quad$ the Mie theory [13];

- $\quad$ the Gans theory [14];

- $\quad$ the discrete-dipole approximation method (DDA) [15];

- the finite-difference time-domain method (FDTD) [16].

\subsubsection{The Mie theory}

The Mie theory is a theoretical approach concerning the optical properties of the nanoparticles. When the nanoparticle dimension is smaller than the wavelength of the 
incident light, such theory predicts that the extinction caused by a metallic nanosphere is estimated in the quasi-static and dipole limit. The relative cross section of the process results:

$$
\sigma_{e x t}=\frac{24 \pi N_{A} a^{3} \varepsilon_{m}^{3 / 2}}{\lambda \ln (10)}\left[\frac{\varepsilon_{i}}{\left(\varepsilon_{r}+\chi \varepsilon_{m}\right)^{2}+\varepsilon_{i}^{2}}\right]
$$

where $N_{A}$ is the surface density, $a$ the radius of the metallic nanosphere, $\varepsilon_{m}$ the dielectric constant of the medium surrounding the nanosphere, $\lambda$ the wavelength, $\varepsilon_{i}$ and $\varepsilon_{r}$ imaginary and real part of the dielectric function of the metallic nanosphere respectively and $\chi$ form factor concerning the nanoparticle. The localized resonance depends also on the interparticle space and on the dielectric constant of the substrate.

\subsubsection{The Gans theory}

The Gans theory extends the Mie theory to spheroidal particles case. In the case of polarization of incident light parallel to the symmetry axis of the spheroid, the cross section of the process is given by:

$$
\sigma_{e x t}=\frac{2 \pi V \varepsilon_{m}^{3 / 2}}{3 \lambda} \sum_{j}\left[\frac{\left(1 / P_{j}\right)^{2} \varepsilon_{j}}{\left(\varepsilon_{r}+\frac{1-P_{j}}{P_{j}} \varepsilon_{m}\right)^{2}+\varepsilon_{j}^{2}}\right]
$$

with $V$ volume of nanoparticle and $P_{j}$ depolarization factors along the three cartesian aces, which hold into account of the anisotropic form of particles.

\subsubsection{The discrete-dipole approximation method (DDA)}

With this method the nanoparticles are divided in a cubic array of $N$ polarizable dipoles, with polarizabilities $\alpha_{i}$ determined by the dielectric function of nanoparticles. The induced dipole $P_{i}$ of every element results $P_{i}=\alpha_{i} E_{l o c, i}$ in an applied plane wave field. The cross section is determined by:

$$
\sigma_{\text {ext }}=\frac{4 \pi k}{\left|\vec{E}_{i n c}\right|^{2}} \sum_{j=1}^{N} \operatorname{Im}\left(\vec{E}_{i n c, j}^{*} \cdot \vec{P}_{j}\right)
$$

with $k=\omega / c$ (in vacuum). Such method can be applied for the calculation of the absorption, scattering, extinction and other optical properties of nanoparticles of various forms and dimensions. It is considered one of the most important methods for the understanding of the structural characteristics and optical properties of nanomaterials and nanostructures. 


\subsubsection{The finite-difference time-domain method (FDTD)}

This is a method of numerical calculation in order to resolve the Maxwell equations directly in the time domain. Being a time-domain method, the solutions can cover a wide frequency range with a single process of simulation. It is a versatile and useful technique in applications where the resonance frequencies are not exactly known. A great variety of magnetic and dielectric materials can be modelled in relatively simple way with such method [17].

\section{Linear response theory: a new interesting idea}

We start considering a system with an hamiltonian of the form:

$$
H=H_{0}+H_{1}
$$

with $H_{1}$ having small effects with respect to $H_{0}$, and of the form:

$$
H_{1}=\lambda A e^{-i \omega t} e^{\eta t}
$$

being $\lambda$ a real quantity and $\eta$ positive, so that in remote past the perturbation is negligible (adiabatic representation: $\lim _{t \rightarrow-\infty} H_{1}=0$ ). In the case of an electric field of frequency $\omega$ we have:

$$
H_{1}=e \vec{E} \cdot \vec{r}
$$

If the electric field is constant in space and its evolution depends on time, it is writable as follows:

$$
\vec{E}=\vec{E}_{0} e^{i \omega t}
$$

The time dependent corresponding current is:

$$
\vec{J}(t)=\sigma(\omega) \vec{E}(t)
$$

The conductivity $\sigma(\omega)$ is in general a complex function of the frequency $\omega$ and can be deduced from linear response theory. Following the standard time-dependent approach, it is possible to find a general formula for the linear response of a dipole moment density $\vec{B}=e \vec{r} / V$ in the $\beta$ direction with the electric field $\vec{E}$ in the $\alpha$ direction, where $V$ is the volume of the system. This permits to deduce the susceptivity $\chi(\omega)$, which is correlated to $\sigma(\omega)$ through the relation:

$$
1+4 \pi \chi(\omega)=1+4 \pi i \frac{\sigma(\omega)}{\omega}
$$


Analytical calculations permit to write the real part of the complex conductivity $\sigma(\omega)$ as:

$$
\operatorname{Re} \sigma_{\beta \alpha}(\omega)=\frac{e^{2} \omega \pi}{V \hbar} S_{\beta \alpha}(\omega)\left(1-e^{-\hbar \omega / K T}\right)
$$

with:

$$
S_{\beta \alpha}(\omega)=\int_{-\infty}^{+\infty} d t\left\langle\vec{r}^{\alpha}(0) \vec{r}^{\beta}(t)\right\rangle_{T} e^{-i \omega t}
$$

The part $\langle\ldots . . .\rangle_{T}$ in the integral (24) is the thermal average, and the exponential factor arises from equilibrium thermal weights for Fermi particles. By considering the identity

$$
v=\frac{d}{d t} r=\frac{i}{\hbar}[H, r]
$$

Eq. (23) can be written in a form containing the velocities correlation function instead of the position correlation function. Assuming the high temperature limit $\hbar \omega<<K T$ (valid in such contests), we obtain:

$$
\operatorname{Re} \sigma_{\beta \alpha}(\omega)=\frac{e^{2}}{2 V K T} \int_{-\infty}^{+\infty} d t\left\langle\vec{v}^{\alpha}(0) \vec{v}^{\beta}(t)\right\rangle_{T} e^{-i \omega t}
$$

The integral in Eq. (25) spans the entire $t$ axis, so we can perform the complete inverse Fourier transform of this equation. It gives:

$$
\left\langle\vec{v}^{\alpha}(0) \vec{v}^{\beta}(t)>_{T}=\frac{K T V}{\pi e^{2}} \int_{-\infty}^{+\infty} d \omega \operatorname{Re} \sigma_{\beta \alpha}(\omega) e^{i \omega t}\right.
$$

The new introduced key idea is the possibility to perform a complete inversion of Eq. (26) on temporal scale, i.e. considering the entire time axis $(-\infty,+\infty)$, not the half time axis $(0,+\infty)$, as usually considered in literature $[9,18]$. This idea is viable if we consider the real part of the complex conductivity $\sigma(\omega)$. Via contour integration by the residue theorem in Eq. (26), the integral is determined by the poles of $\operatorname{Re} \sigma(\omega)$. This leads to an exact formulation and gives a powerful method to describe the velocities correlation function (and consequently the mean square displacement and the diffusion coefficient) in analytical way.

\section{The other important functions in the nano-bio-context}

Another interesting quantity at nanolevel is the mean squared displacement of particles at equilibrium, defined as:

$$
R^{2}(t)=\left\langle[\vec{R}(t)-\vec{R}(0)]^{2}\right\rangle
$$


where $R(t)$ is the position vector at time $t$. Through a coordinate transformation relative to the integration region it is possible to rewrite the relation (27) as follows:

$$
R^{2}(t)=2 \int_{0}^{t} d t^{\prime}\left(t-t^{\prime}\right)\left\langle\vec{v}\left(t^{\prime}\right) \cdot \vec{v}(0)\right\rangle
$$

The mean squared displacement can therefore be evaluated through the velocities correlation function. Through Eq. (26) it is possible to deduce also the diffusion coefficient $D$ :

$$
D(t)=\frac{d R^{2}(t)}{2 d t}=\int_{0}^{t} d t^{\prime}\left\langle\vec{v}\left(t^{\prime}\right) \cdot \vec{v}(0)\right\rangle
$$

The three relations (26), (28), (29) are fundamental in deducing the most important characteristics concerning the transport phenomena.

\section{About the complex conductivity $\sigma(\omega)$}

Let us consider a particle in a region in which there is an electric time-oscillating field, direct along $z$-axis, with an elastic-type and a friction-type force acting on system; the dynamic equation of the particle can be written as:

$$
m \ddot{z}=-k z-\lambda \dot{z}+e E_{o} e^{-i \omega t}
$$

where $m$ is the mass of the particle, $k$ the strenght constant of the oscillators, $\lambda=m / \tau$ and $\tau$ is the relaxation time. We consider solutions of the form:

$$
z(t)=z_{0} e^{-i \omega t}
$$

The current density in the field direction is:

$$
j_{z}=n e \dot{z}=n e z_{0}(-i \omega) e^{i \omega t}=\sigma E
$$

From Eq. (32), via analytical calculations, it is possible to obtain the frequency dependent complex conductivity:

$$
\sigma=\frac{i \omega n e^{2}}{m\left(\omega^{2}-\omega_{0}^{2}+i \lambda \omega\right)}
$$

The real part of the complex conducivity (33) results [1,19]:

$$
\operatorname{Re} \sigma(\omega)=\frac{\tau n e^{2}}{m} \frac{\omega^{2} / \tau^{2}}{\left(\omega_{0}^{2}-\omega^{2}\right)^{2}+\omega^{2} / \tau^{2}}
$$




\section{About the Drude-Lorentz-like models: a new promising "plasmon model"}

Recently it has been published a new formulation of the Drude-Lorentz model [1,20], based on linear response theory and resonant plasmonic mode; it is able to accommodate some of the observed departures and gives a detailed description of the dynamic response of the carriers at nano-level. One of the peculiarities of this new model is the inversion of relation (26), extending the integration on time to the entire time axis $(-\infty,+\infty)$; this procedure is not trivial and it was introduced for the first time in the nano-bio-context. One of the main advantages is the possibility to obtain analytical relations for the description of the dynamic behaviour of nanosystems. Such relations are functions of parameters, experimentally obtainable through Time-resolved techniques. An important consequence of the application of such formulation turns out to be the mathematical justification of the unexpected experimental results of high initial mobility of charge carriers in devices based on semiconductor nanomaterials, as the dye sensitized solar cells (DSSCs). The increase of the diffusion coefficient implies a raise of the efficiency of such electro-chemical cells. The new formulation is able to describe very adequately the properties of transport of nano-biomaterials, but it holds also for other objects, like ions [19].

Many experimental data have indicated that plasmon models describe nanostructured systems in particularly effective way. At quantum level, the key factors incorporating the quantum behaviour are the weights $f_{i}$, related to plasma frequencies by:

$$
\omega_{p_{i}}{ }^{2}=\frac{4 \pi N e^{2}}{m} f_{i}
$$

where $N$ is the carrier density, $m$ and $e$ respectively the mass and the charge of the electron.

The velocities correlation function in quantum case assumes the form:

$$
\langle\vec{v}(0) \cdot \vec{v}(t)\rangle=\left(\frac{K T}{m}\right) \sum_{i}\left(f_{i} \exp \left(-\frac{t}{2 \tau_{i}}\right)\left[\cos \left(\frac{\alpha_{i R}}{2} \frac{t}{\tau_{i}}\right)-\frac{1}{\alpha_{i R}} \sin \left(\frac{\alpha_{i R}}{2} \frac{t}{\tau_{i}}\right)\right]\right)
$$

with:

$$
\alpha_{i R}=\sqrt{4 \tau_{i}^{2} \omega_{i}^{2}-1}
$$

$$
\langle\vec{v}(0) \cdot \vec{v}(t)\rangle=\frac{1}{2}\left(\frac{K T}{m}\right) \sum_{i}\left(\left(\frac{f_{i}}{\alpha_{i I}}\right)\left[\left(1+\alpha_{i I}\right) \exp \left(-\frac{\left(1+\alpha_{i I}\right)}{2} \frac{t}{\tau_{i}}\right)-\left(1-\alpha_{i I}\right) \exp \left(-\frac{\left(1-\alpha_{i I}\right)}{2} \frac{t}{\tau_{i}}\right)\right]\right)
$$

with:

$$
\alpha_{i I}=\sqrt{1-4 \tau_{i}^{2} \omega_{i}^{2}}
$$

( $K$ is the Boltzmann's constant, $T$ the temperature of the system, $\omega_{i}$ and $\tau_{i}$ frequencies and decaying times of each mode respectively). 
Integrating these expressions through Eqs. (28) and (29), we obtain the mean squared displacement $R^{2}$ and the diffusion coefficient $D[20,21]$.

\section{Some interesting results of the new model}

The model has demonstrated at today high generality and good accordance with experiments. It has been applied in relation to the most commonly used and studied materials at nano-level, i.e. zinc oxide $(\mathrm{ZnO})$, titanium dioxide $\left(\mathrm{TiO}_{2}\right)$, gallium arsenide $(\mathrm{GaAs})$, silicon ( $\mathrm{Si})$ and carbon nanotubes $(\mathrm{CN})$, also at nano-bio-sensoristic level [19,22].

In Figure 1 it is showed $R^{2}$ for doped Silicon. For this systems the conductivity is the contribution of a Drude-Lorentz term and a Drude term. At large times the Drude-Lorentz term leads to an $R^{2}$ approaching a constant value (Figure 2), while the Drude term alone is the dominant one. Therefore, for sufficiently large times, only the Drude term survives.

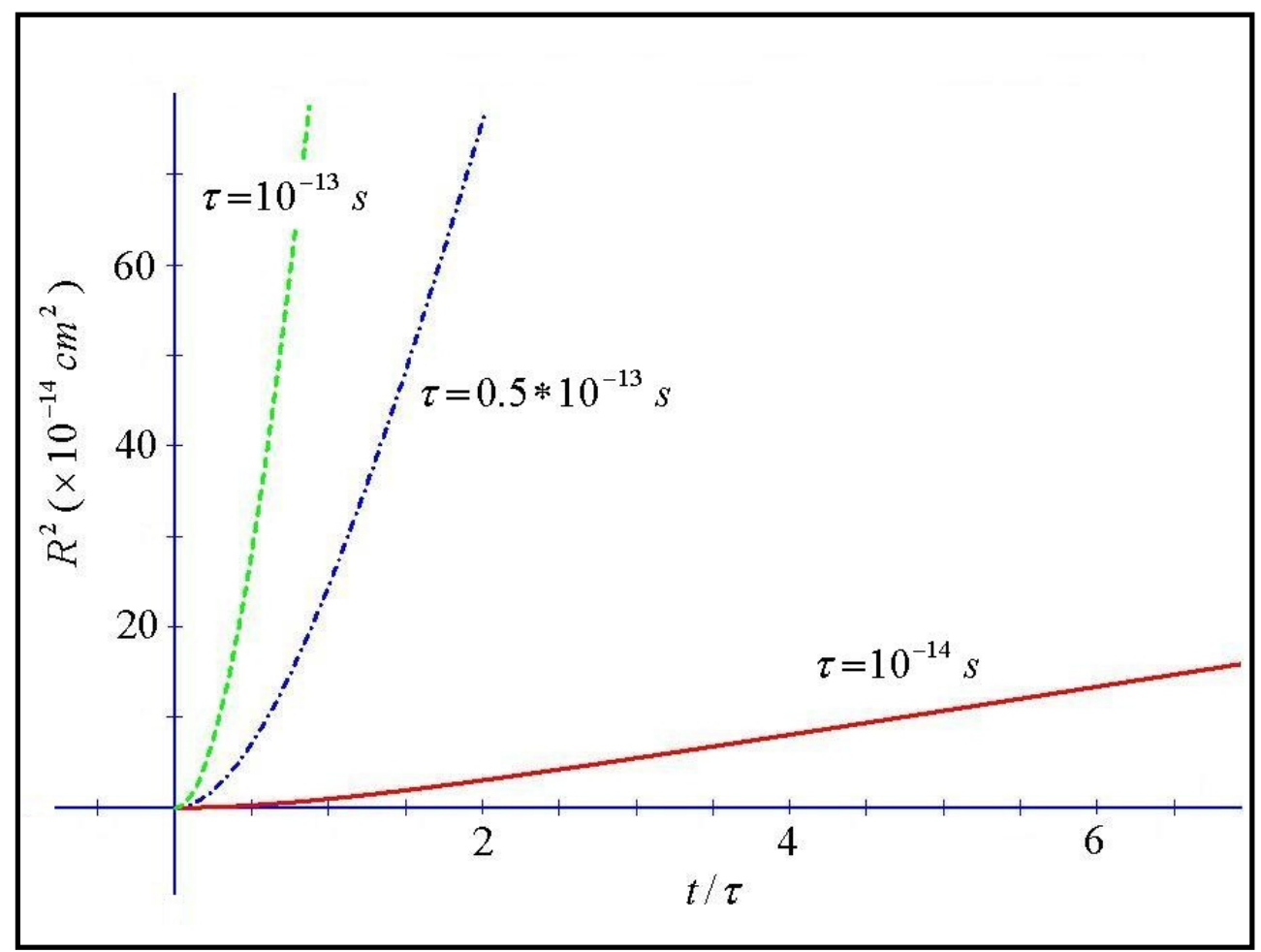

Figure 1. $R^{2}$ vs $x=t / \tau$ for some representative values of $\tau$, typical of doped Silicon [23] $\left(\omega_{0}=0\right.$, $T=300 \mathrm{~K}$ ) (Drude model). A complete description of $R^{2}$ for Silicon requires the evaluation of the contribution of the Drude-Lorentz part.

We can observe that the linear relation at large times becomes quadratic at smaller times. The cross-over between the two regimes occurs at times comparable to the scattering time. 
This means that diffusion occurs after sufficient time has elapsed, so that scattering events become significant, while at smaller times the motion is essentially ballistic.

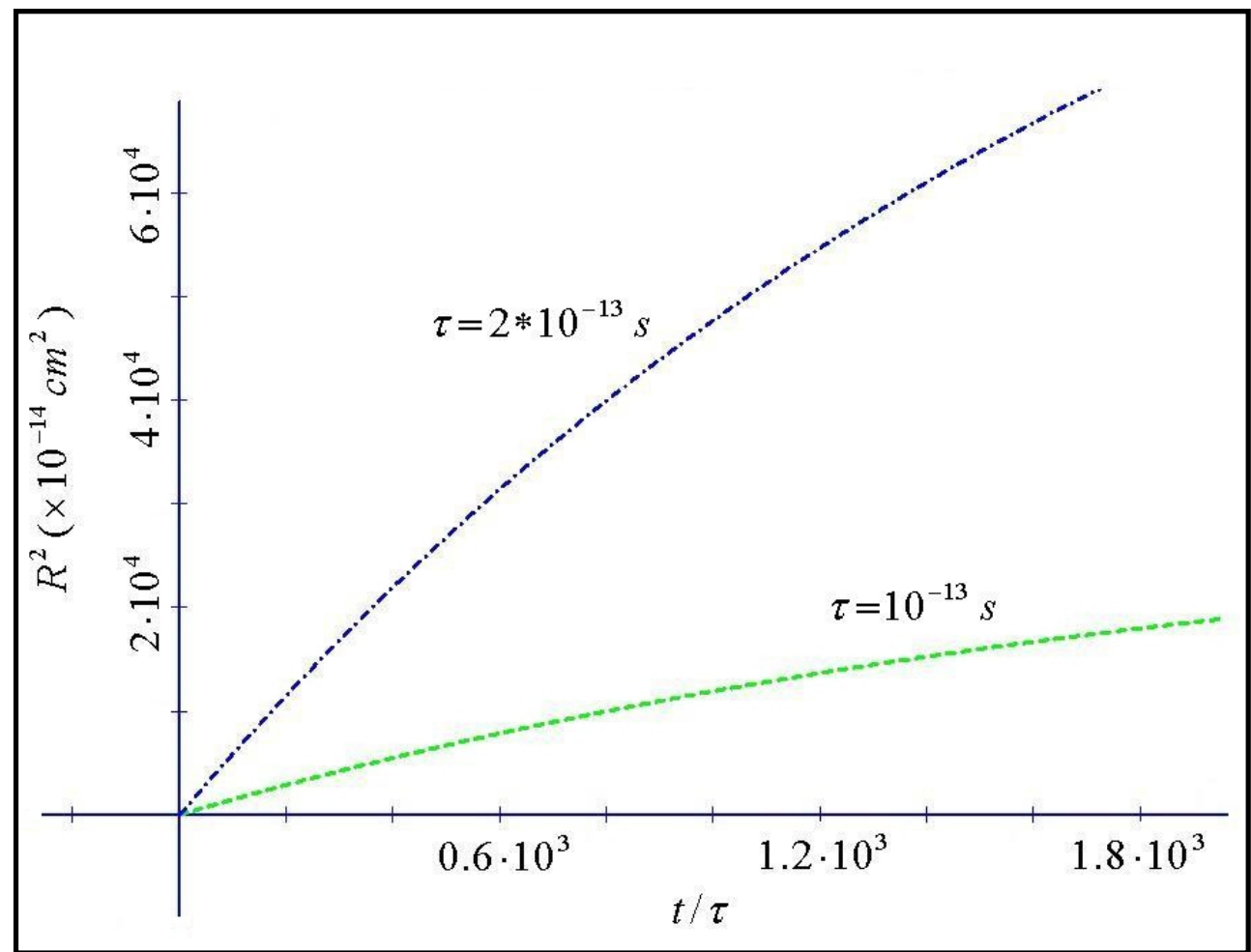

Figure 2. $R^{2}$ vs $x=t / \tau$ for 2 values of $\tau\left(\omega_{0}=1.12 \cdot 10^{11} \mathrm{~Hz}\right.$ dot-dashed line; $\omega_{0}=2.24 \cdot 10^{11} \mathrm{~Hz}$ dashed line) for $\mathrm{TiO}_{2}(m=6 m e, T=300 \mathrm{~K})$. Saturation values occur at sufficiently large $t$.

The Drude behaviour is contrasted by the plasmon behaviour. It has reported representative cases at large times $x=t / \tau \gg>1$ (Figure 2) for $\mathrm{TiO}_{2}$ nanoparticle films with small frequencies $\omega_{0}$, i.e. close to the Drude case. It is observable that all of the curves reach a plateau value at sufficiently long times and that the slope within a given time interval increases with $\tau$. As consequences of this behaviour, the plateau of $R^{2}$ may become larger than the size of the nanoparticles composing the films, depending on the parameters $\omega_{0}$ and $\tau$, indicating that carriers created at time $t=0$ will have enhanced mobility in the nanoporous films at small times, on the order of few $\tau$, in contrast with a commonly expected low mobility in the disordered $\mathrm{TiO}_{2}$ network. Secondly, the time derivative of $R^{2}$ decreases to zero at very large times, indicating localization by scattering and therefore leading to decreasing mobility and to the existence of a strictly insulating state for $t \rightarrow \infty$

Few cases corresponding to short time behaviour and large frequencies are showed in Figure 3, with the scattering time in the range $10^{-13}-10^{-14} \mathrm{~s}$. 


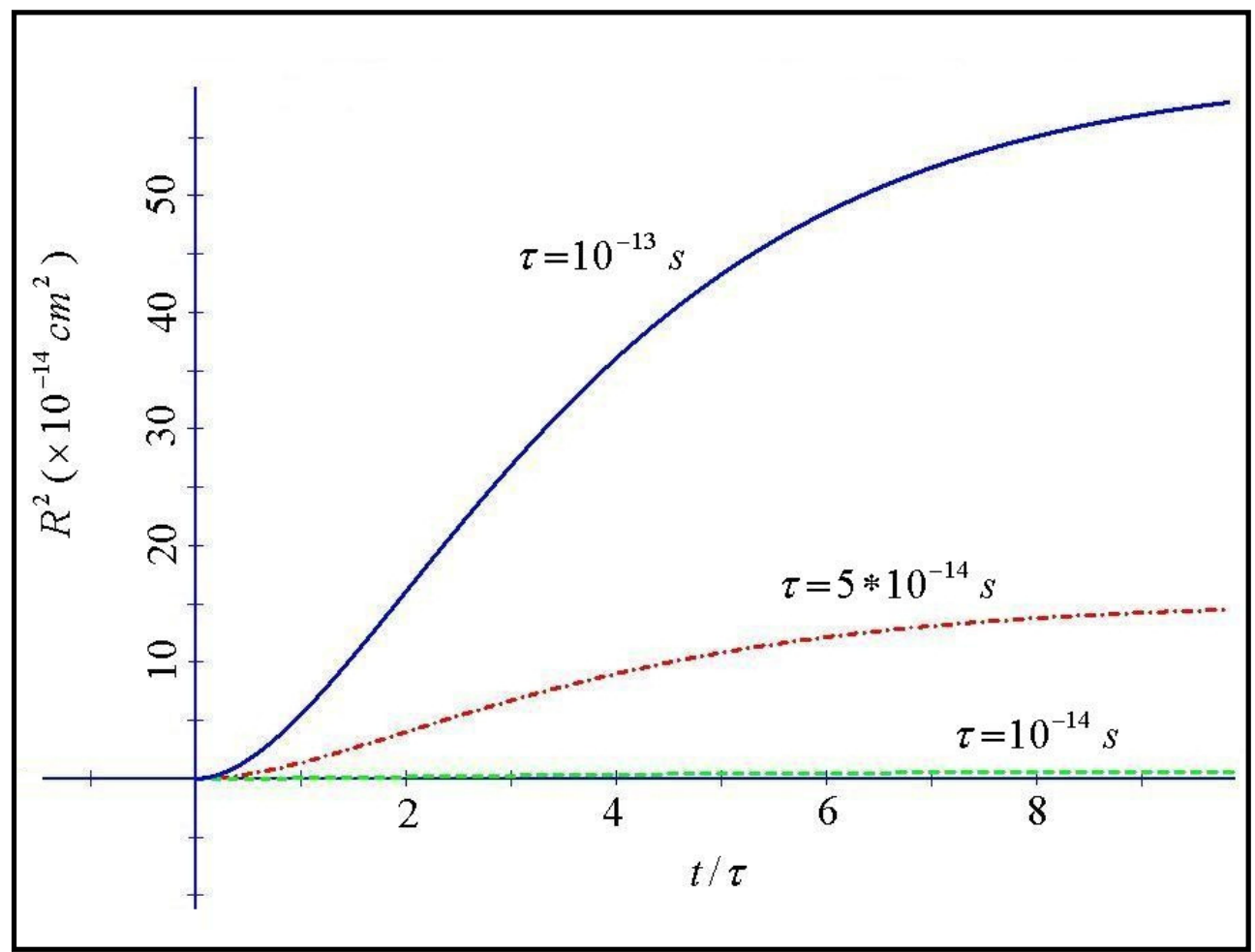

Figure 3. $R^{2}$ vs $x=t / \tau$ for 3 values of $\tau\left(\omega_{0}=0.5 \cdot 10^{13} \mathrm{~Hz}\right.$ solid line; $\omega_{0}=10^{13} \mathrm{~Hz}$ dot-dashed line; $\omega_{0}=0.5 \cdot 10^{14} \mathrm{~Hz}$ dashed line) for $\mathrm{TiO}_{2}(m=6 m \mathrm{e}, \mathrm{T}=300 \mathrm{~K})$.

In relation to the behaviour of the velocities correlation function, excluding the Drude model $\left(\omega_{0}=0\right)$, characterized by simple exponential decreasing functions with time, the correlation function of velocities corresponds to either a damped oscillatory behaviour (Eq. (36)), or to a superposition of two exponentials (Eq. (38)), with quite different decay times depending on the value of $\alpha_{I}$ (Figures 4,5 ).

At $\alpha \cong 1$, i.e. close to Drude behaviour, proper case of some systems [3], the current results the superposition of a short $\approx \tau$ and long $\tau /\left(1-\alpha_{I}\right)$ time decay modes. The current is a damped oscillating function of time (Eq. (36)) when $\omega_{0} \tau \gg>1$, and will have double exponential behaviour for $\omega_{0} \tau<<1$ (Eq. (38)).

From this analysis, it is viable the possibility that the previous results can give an explanation of the ultra-short times and high mobilities, with which the charges spread in mesoporous systems, of large interest in photocatalitic and photovoltaic systems [24]; the relative short times (few $\tau$ ), with which charges can reach much larger distances than typical dimensions of nanoparticles, indicate easy charges diffusion inside the nanoparticles. The unexplained fact, experimentally found, of ultrashort injection of charge carriers (particularly in Grätzel's cells) can be related to this phenomenon. 


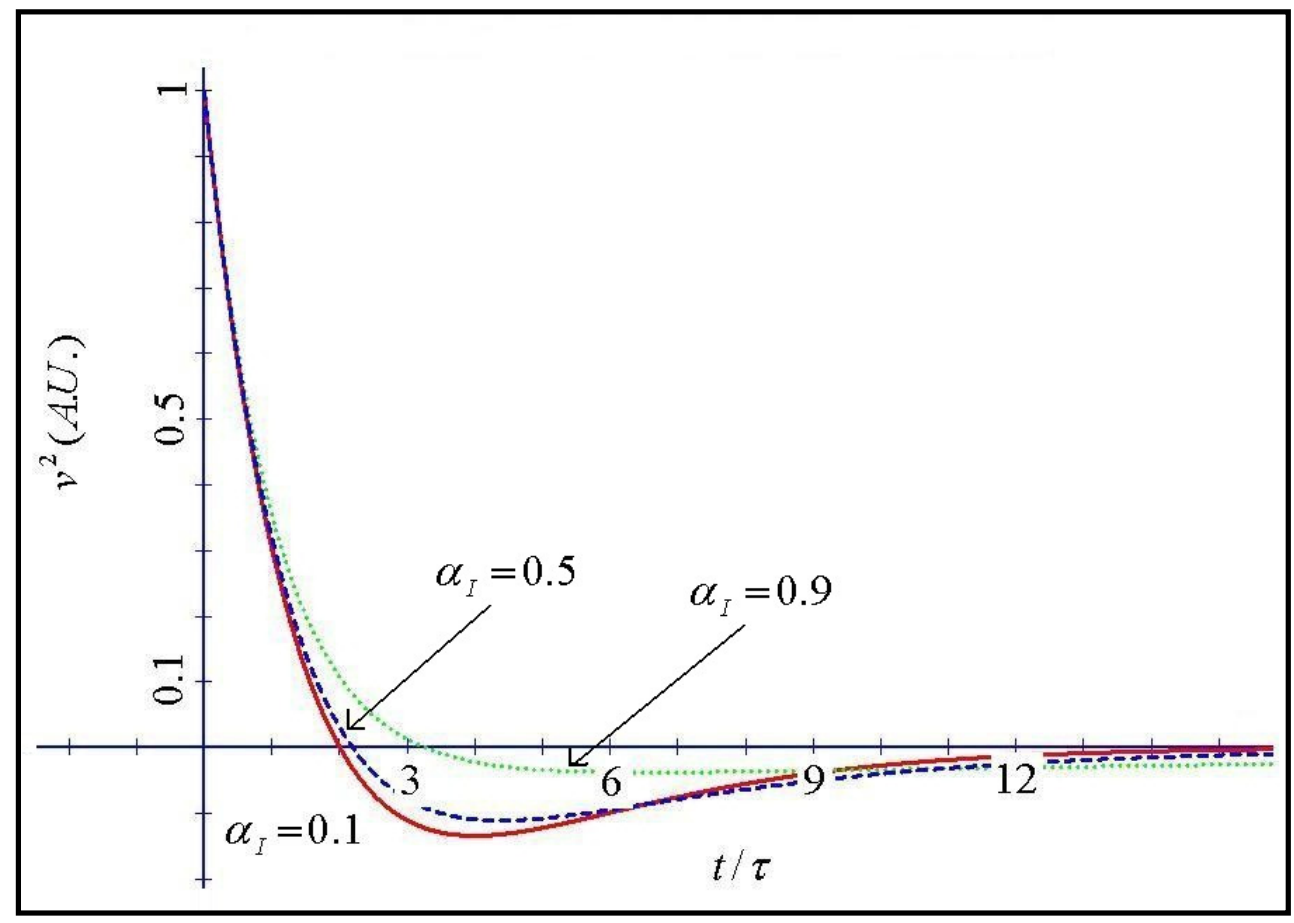

Figure 4. Velocities correlation function vs $x=t / \tau$ for some values of $\alpha_{I}(m=6 m e, T=300 K)$.

In systems with charge localization, where the carrier mean free path is comparable to the characteristic dimension of the nanoparticles, the conductivity response becomes more complicated. The response at low frequencies is characterized by an increasing real part of the conductivity and by a negative imaginary part. Deviations from the Drude model become strong in nanostructured materials, such as photoexcited $\mathrm{TiO}_{2}$ nanoparticles, $\mathrm{ZnO}$ films, InP nanoparticles [25], semiconducting polymer molecules [26], and carbon nanotubes [27].

In isolated $\mathrm{GaAs}$ nanowires the electronic response exhibits a pronounced surface plasmon mode, that forms within $300 \mathrm{fs}$, before decaying within $10 \mathrm{ps}$ as a result of charge trapping at the nanowire surface. The conductivity in this case was fitted by using the Drude model for a plasmon and the mobility found to be remarkably high, being roughly one-third of that typical for bulk GaAs at room temperature.

The Smith model with $c_{1}=-1$ is obtained as a limit of the new introduced plasmon model when $\alpha_{I} \rightarrow 0$. On performing this limit in Eq. (38) one finds the expression obtained by Smith with a scattering time twice the plasmon scattering time $\tau$. The situation $\alpha_{I} \rightarrow 0$ corresponds to $\tau \approx 1 / 2 \omega_{0}$. From the other hand, both Smith's and the new model reduce to the Drude model in the limit $\omega_{o} \rightarrow 0$. So, although the two models are analytically different, their predictions are expected to be quite similar. From Figure 4 it is possible to note that, for 
$\alpha_{I} \rightarrow 0$, the lower curve is of the form of the Smith curve. The backscattering mechanism invoked by Smith arises in a natural way in this new model, without further assumptions on successive scattering events.

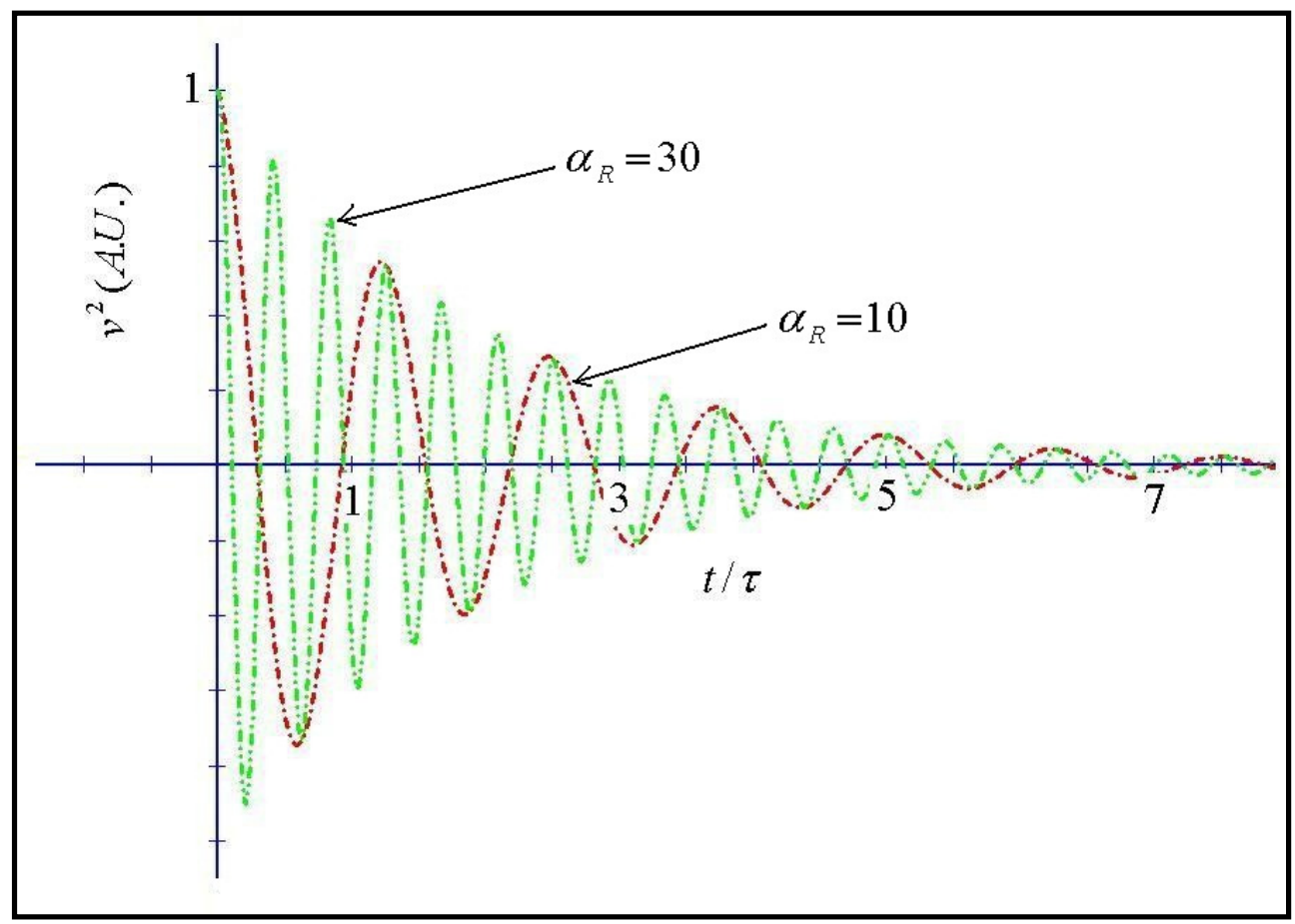

Figure 5. Velocities correlation function vs $x=t / \tau$ for two values of $\alpha_{R}\left(m=6 m_{\mathrm{e}}, T=300 \mathrm{~K}\right)$. Evident exponentially damped oscillations are displayed in this case.

Time evolution of charges can be systematically studied by means of time resolved techniques. The $\mathrm{THz}$ technique allows a detailed investigation of very short time behaviour, such as the photoinjection, as well as longer time behaviour such as thermalized motion. The studies have indicated a common mechanism of the short time domain in nanostructures, where carriers are close to Drude behaviour with a rather large diffusion coefficient, followed by a range with time decreasing mobility. This latter stage is characterized by decay of the response as the superposition of a short time and a longer time exponentials.

The time response of $\mathrm{ZnO}$ films, nanowires, and nanoparticles to near-UV photoexcitation has been investigated in THz experiments. Films and nanoparticles show ultrafast injection, but with the addition of a second slower component. For $\mathrm{ZnO}$ nanoparticles, double exponential decay indicates characteristic times $\tau_{1} \approx 94 p s$ and $\tau_{2} \approx 2.4 n s$. From Eq. (38) it is possible to deduce a ratio of two different relaxation times $\tau_{1}$ and $\tau_{2}$; it is $\tau_{1} / \tau_{2}=\left(1-\alpha_{I}\right) /\left(1+\alpha_{I}\right)$. Therefore, on using the experimental values, we find $\alpha_{I}=0.92$, 
from which we deduce $\omega_{0} \tau \cong 0.2$. For $\tau$ of the order of $10^{-13} \mathrm{~s}$, we obtain $\omega_{0} \cong 1.5 \cdot 10^{12} \mathrm{~Hz}$, which is of the correct order of magnitude for the resonance in the infrared. The same procedure for injection times of films (500 nm grains) leads to $\omega_{0} \cong 2.5 \cdot 10^{12} \mathrm{~Hz}$ (for $\tau \cong 10^{-13}$ s ).

In GaAs nanowires $[27,28]$ a resonance at $\omega_{o}=0.3-0.5 \mathrm{THz}$ has been suggested to explain the frequency-dependent conductivity obtained from $\mathrm{THz}$ experiments. A long characteristic obtained time $\tau_{c} \approx 1.1 \div 5 \cdot 10^{-12} \mathrm{~s}$ is in accordance with the new model, corresponding to $\tau_{c}=\tau /\left(1-\alpha_{I}\right)=5.10^{-12} s$ and leading to $\alpha_{I}=0.1 \div 0.8$ for $\tau=10^{-12} s$ and $\omega_{0} \tau=0.3 \div 0.49$.

Similar considerations can be applied to single walled carbon nanotubes (SWCN), where the resonance state is at $\omega_{o}=0.5 \mathrm{THz}$ with Drude-Lorentz scattering time $\tau \approx 10^{-13} \mathrm{~s}$ and to sensitized $\mathrm{TiO}_{2}$ nanostructured films. In the latter the conductivity of electrons injected from the excited state of the dye molecule into the conduction band of $\mathrm{TiO}_{2}$ is initially more Drude-like, and then evolves into a conduction dominated by strong backscattering as the carriers equilibrate with the lattice. These results conform to the backscattering mechanism of the new plasmon model.

In Figure 6 it is presented the fitting of data of GaAs photoconductivity $[27,28]$ with the new model.

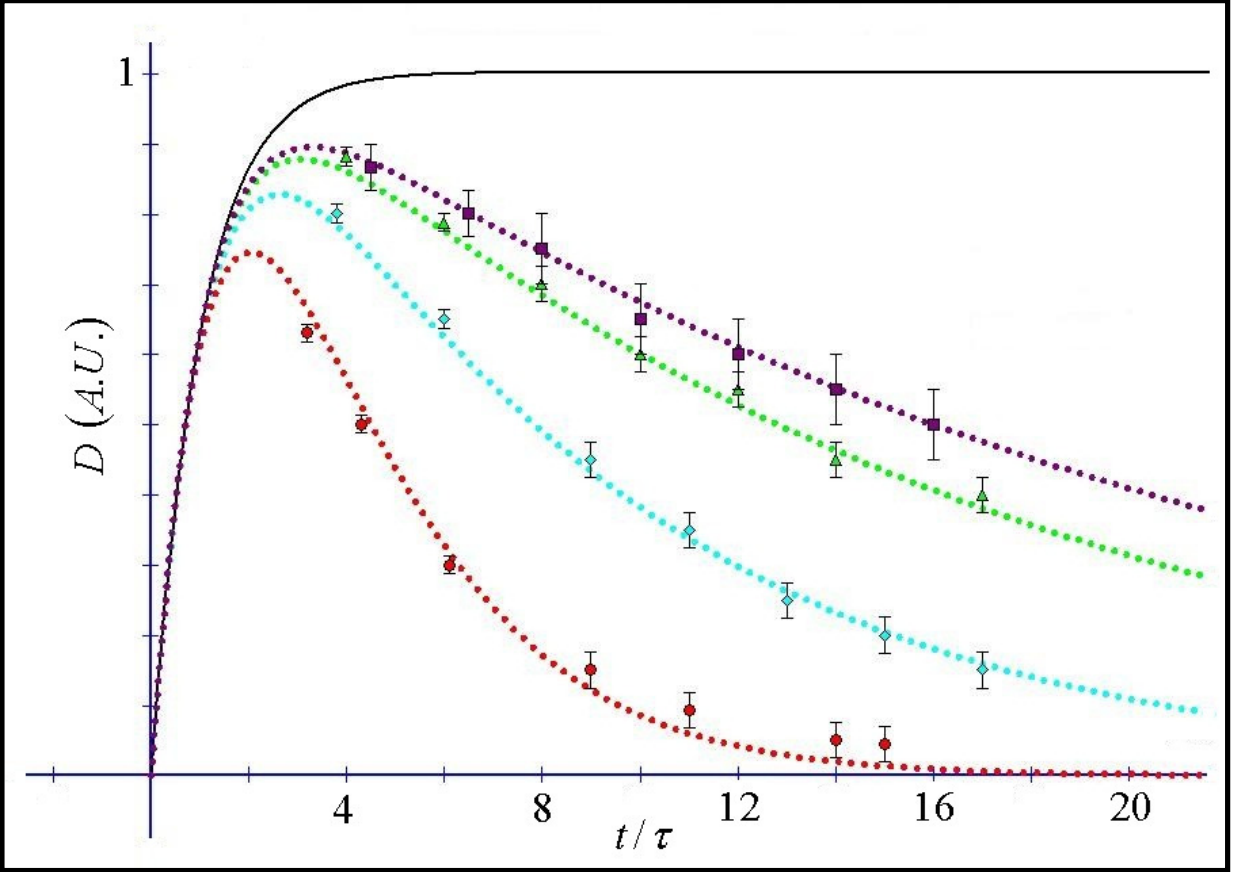

Figure 6. Behaviour of the diffusion coefficient $D$ for $\alpha_{I}$ (Eq. (39) at classical level [1]) varying in the interval [0.1-1] and $\tau=10^{-12} \mathrm{~s}$. Note that $D \rightarrow 0$ for $t \rightarrow \infty$, indicating absence of diffusion at very long times. 
In this figure the upper curve is the Drude result corresponding to $\omega_{0}=0$. It is the highest value of the diffusion coefficient, as $\omega_{0}$ varies from zero to its maximum value. The dots in Figure 6 are experimental data derived from $\mathrm{THz}$ spectroscopy, the error bars on dots represent the distribution of the experimental data and the lines the predictions of the new plasmon model. $D$ tends to a vanishing value as $t \rightarrow \infty$, the larger $\omega_{0}$ the faster this vanishing occurs. The results can be interpreted as follows: at early time, of the order of $\tau$, the system behaves as Drude-like, irrespective of $\omega_{0}$, with carriers assuming large mobility values (the numerical evaluation gives $D \approx 7.5 \mathrm{~cm}^{2} / \mathrm{s}$ for the case of Figure 6); at increased times, charges become progressively localized as a result of scattering, significantly in agreement with the conclusions drawn by THz spectroscopy $[29,30]$.

\section{Conclusions}

It is hoped that this new plasmon model will be useful to describe experimental data as an alternative to other generalizations of the Drude model. The principal findings of it are the reversal of the current in small systems like nanoparticles and the time dependence of the transport parameter $D$, which describes the dynamics of the system at short and long time, with increased mobilities at very short times followed by localization at longer times. This unusual behaviour, with respect to the predicted Drude-like, converges as a whole in the peculiar frequency dependence of the optical conductivity. The strength of the new model consist of its ability to accommodate this behaviour and include previous models, like the Smith model [19].

\section{Author details}

Paolo Di Sia

Free University of Bozen-Bolzano, Bruneck-Brunico (BK), Italy

\section{References}

[1] Di Sia P. An Analytical Transport Model for Nanomaterials. Journal of Computational and Theoretical Nanoscience 2011; 8: 84-89.

[2] Wolf EL. Nanophysics and Nanotechnology: An Introduction to Modern Concepts in Nanoscience. John Wiley \& Sons; 2006.

[3] Schmuttenmaer CA. Using Terahertz Spectroscopy to Study Nanomaterials. Terahertz Science and Technology 2008; 1(1): 1-8.

[4] Ziman M. Principles of the Theory of Solids. Cambridge University Press; 1979.

[5] Kittel C. Introduction to Solid State Physics. Wiley New York; 1995.

[6] Levy O, Stroud D. Maxwell Garnett theory for mixtures of anisotropic inclusions: Application to conducting polymers. Physical Review B 1997; 56(13): 8035-8046.

[7] Han J, Zhang W, Chen W, Ray S, Zhang J, He M, Azad AK, Zhu Z. Terahertz Dielectric Properties and Low-Frequency Phonon Resonances of $\mathrm{ZnO}$ Nanostructures. The Journal of Physical Chemistry C 2007; 111(35): 13000-13006. 
[8] Han JG, Wan F, Zhu ZY, Liao Y, Ji T, Ge M, Zhang ZY. Shift in low-frequency vibrational spectra of transition-metal zirconium compounds. Applied Physics Letters 2005; 87(17): 172107-172109.

[9] Smith NV. Classical generalization of the Drude formula for the optical conductivity. Physical Review B 2001; 64(15): 155106-155111.

[10] Shipway AN, Katz E, Willner I. Nanoparticle arrays on surfaces for electronic, optical and sensoric applications. ChemPhysChem 2000; 1: 18-52.

[11] Hutter E, Fendler JH. Exploitation of Localized Surface Resonance. Advanced Materials 2004; 16(19): 1685-1706.

[12] Willets KA, Van Duyne RP. Localized Surface Plasmon Resonance Spectroscopy and Sensing. Annual Review of Physical Chemistry 2007; 58: 267-297.

[13] Stuart DA, Haes AJ, Yonzon CR, Hicks EM, Van Duyne RP. Biological applications of localised surface plasmonic Phenomenae. IEE Proceedings-Nanobiotechnology 2005; 152(1): 13-32.

[14] Gulati A, Liao H, Hafner JH. Monitoring Gold Nanorod Synthesis by Localized Surface Plasmon Resonance. The Journal of Physical Chemistry B 2006; 110(45): 22323-22327.

[15] Zhang ZY, Zhao Y-P. Optical properties of helical Ag nanostructures calculated by discrete dipole approximation method. Applied Physics Letters 2007; 90: 221501-221503.

[16] Sherry LJ, Chang S-H, Schatz GC, Van Duyne RP. Localized Surface Plasmon Resonance Spectroscopy of Single Silver Nanocubes. Nano Letters. 2005; 5(10): 20342038.

[17] Zhang W, Yue Z, Wang C, Yang S, Niu W, Liu G. Theoretical models, Fabrications and Applications of Localized Surface Plasmon Resonance sensors. 978-1-4244-49644/10/\$25.00@2010 IEEE 2010; pp. 4; Project supported by the National Natural Science Foundation of China (Grant N. 60574091, 60871028).

[18] Sassella A, Borghesi A, Pivac B, Pavesi L. Characterization of porous silicon by microscopic Fourier transform infrared spectroscopy; 266-267. 9 $9^{\text {th }}$ International Conference on Fourier transform spectroscopy; 1994.

[19] Di Sia P. Classical and quantum transport processes in nano-bio-structures: a new theoretical model and applications. PhD Thesis. Verona University - Italy; 2011.

[20] Di Sia P. An Analytical Transport Model for Nanomaterials: The Quantum Version. Journal of Computational and Theoretical Nanoscience 2012; 9: 31-34.

[21] Di Sia P. Oscillating velocity and enhanced diffusivity of nanosystems from a new quantum transport model. Journal of Nano Research 2011; 16: 49-54.

[22] Di Sia P. New theoretical results for high diffusive nanosensors based on $\mathrm{ZnO}$ oxides. Sensors and Transducers Journal 2010; 122(1): 1-8.

[23] Pirozhenko I, Lambrecht A. Influence of slab thickness on the Casimir force. Physical Review A 2008; 77: 013811-013818.

[24] Grätzel M. Solar Energy Conversion by Dye-Sensitized Photovoltaic Cells. Inorganic Chemistry 2005; 44(20): 6841-6851.

[25] Nienhuys H-K, Sundström V. Influence of plasmons on terahertz conductivity measurements. Applied Physics Letters 2005; 87: 012101-012103. 
[26] Hendry E, Koeberg M, Schins JM, Nienhuys HK, Sundström V, Siebbeles LDA, Bonn M. Interchain effects in the ultrafast photophysics of a semiconducting polymer: $\mathrm{THz}$ timedomain spectroscopy of thin films and isolated chains in solution. Physical Review B 2005; 71: 125201-125210.

[27] Parkinson P, Lloyd-Hughes J, Gao Q, Tan HH, Jagadish C, Johnston MB, Herz LM. Transient Terahertz Conductivity of GaAs Nanowires. Nano Letters 2007; 7(7): 21622165.

[28] Parkinson P, Joyce HJ, Gao Q, Tan HH, Zhang X, Zou J, Jagadish C, Herz LM, Johnston MB. Carrier Lifetime and Mobility Enhancement in Nearly Defect-Free Core-Shell Nanowires Measured Using Time-Resolved Terahertz Spectroscopy. Nano Letters 2009; 9(9): 3349-3353.

[29] Di Sia P, Dallacasa V, Dallacasa F. Transient conductivity in nanostructured films. Journal of Nanoscience and Nanotechnology 2011; 11: 1-6.

[30] Di Sia P, Dallacasa V. Anomalous charge transport: a new "time domain" generalization of the Drude model. Plasmonics 2011; 6(1): 99-104. 